\title{
Tres décadas de una revista de Historia: La Tercera Serie del Boletín del Instituto Ravignani, 1989-2020
}

Alejandro Cattaruzza

Instituto de Historia Argentina y Americana "Dr. E. Ravignani", Universidad de Buenos Aires/ CONICET, Argentina.

Correo electrónico: cattaale@yahoo.com.ar

Artículo recibido: 24 de diciembre de 2020

Aprobación final: 19 de febrero de 2021

\section{Resumen}

Este artículo está dedicado a analizar las reorientaciones y las permanencias registradas en las perspectivas, las áreas temáticas y los períodos asumidos en los trabajos publicados en la Tercera Serie del Boletín. Se ponen en relación estas tendencias con fenómenos ocurridos en el propio instituto, así como en la base universitaria y en la de investigación en la que está inserto. A su vez, los cambios impulsados por la indización y la edición virtual han sido tomados particularmente en cuenta. Se trata, entonces, de un intento de examen de ciertos ámbitos de la producción historiográfica argentina en tiempos recientes.

Palabras clave: Historiografía argentina reciente, Revistas universitarias de historia,Instituto Ravignani.

Three Decades of a History Review: the Third Series of the Boletín del Instituto Ravignani, 1989-2020

\begin{abstract}
This article analyses the perspectives, the areas and the periods assumed by the authors whose works were published in the Boletín del Instituto Ravignani's Third Series (1989-2020). Those trends are linked with diverse processes that took place in the Instituto Ravignani, but also in the university and in the research system on which it depends. At the same time, the changes caused by the indexation
\end{abstract}

1 Deseo agradecer a Julio Djenderedjian y a Juan Luis Martirén tanto el aporte de datos como el esfuerzo realizado en la tarea de edición. 
and online publication of the review are especially attended. It is, therefore, an approach to the study of certain areas of the recent Argentine historiography.

Keywords: Recent Argentine Historiography, Academic History Reviews, Instituto Ravignani.

\section{Miradas sobre las revistas de historia}

Desde hace tiempo, las revistas de historia fueron examinadas con objetivos diversos y de distintos modos por quienes se dedicaron a indagarlas. Si bien algunos trabajos, y tramos de trabajos, estuvieron referidos a las revistas llamadas de divulgación, que en ciertos contextos han exhibido éxitos de ventas, permanencia, dinamismo y a veces vínculos estrechos con zonas de la historia universitaria, buena parte de aquella producción estuvo dedicada al análisis de otro tipo de publicaciones: las que usualmente se denominan revistas académicas, científicas, especializadas o de investigación. Estos términos, según argumentan Susana Romanos de Tiratel y Nora López, suelen aplicarse a las que Nisonger designa como revistas científicas, cuyas características serían las que siguen: "los autores son expertos que escriben en un estilo académico para una audiencia especializada sobre temas de investigación; suele tener un aspecto físico serio, notas al pie de página y bibliografías, un comité editorial, puede contar con evaluadores externos así como estar cubierta por servicios de indización y condensación dedicados a analizar la literatura de distintas disciplinas y tiene una circulación y tiradas limitadas [...]. A esto se puede agregar el tipo de publicador:universidades, asociaciones y colegios profesionales, academias, institutos o centros de investigación y editoriales comerciales dedicadas a la publicación científica" (Romanos de Tiratel y López, 2004: 102). Por otra parte, un parecer extendido hace de esas revistas uno de los más relevantes actores de la comunicación científica. Se trata de un modelo en el cual, cómodamente, puede instalarse el Boletín del Instituto Ravignani en su Tercera Serie.

Con frecuencia, los trabajos dedicados a estos objetos culturales propios del mundo de los historiadores fueron redactados en el marco de la conmemoración de alguna fecha importante para la publicación. Sin que esa práctica haya desaparecido del todo -y este mismo trabajo, como otros que se citarán en aquí, podrían ser vistos como pruebas de su supervivencia-, las publicaciones pasaron paulatinamente a ser concebidas como objetos de estudio que merecían un tratamiento científico, más allá de la celebración.

Sin embargo, ese anhelo de cientificidad no anulaba otras diferencias de perspectiva. Así, tanto en trabajos que se concentraban en una publicación como en los que tomaban un grupo de ellas, las revistas de historia fueron concebidas, por ejemplo, como piezas importantes en la consolidación institucional de la disciplina. Desde ese punto de vista, se desatacó su doble carácter de testimonio de la existencia de un proceso de especialización y profesionalización, y de agente de ese mismo fenómeno; esta nota no era, desde ya, específica de la profesionalización en historia. También se consideró el papel que a menudo cumplieron aquellas 
publicaciones como ámbitos de legitimación para el inicio o la afirmación de trayectorias académicas, así como su condición de herramientas que grupos y sectores se daban para desarrollar sus pujas por la autoridad y el poder dentro del universo historiográfico, en las que aparecía también en juego el prestigio que podía otorgar el publicar en una determinada revista, o formar parte de sus comités de redacción.

Naturalmente, no sólo eran varias las concepciones de base acerca de cuáles eran las funciones que cumplían las publicaciones y cuál su papel en la vida académica, sino también las maneras de abordarlas. Hacia finales de los años setenta del siglo XX y comienzos de la década abierta en 1980, aproximadamente, las tentativas de cuantificación se hicieron frecuentes, en ejemplos que todavía hoy se citan y se leen con beneficio; así ocurre, entre otros, con los trabajos de Corbin, Carbonell y Darnton, mencionados en la bibliografía ${ }^{2}$. Más o menos entusiastas acerca de lo que puede esperarse de una aproximación cuantitativa, quienes la ensayan aún de manera ocasional suelen advertir tanto las dificultades que supone la construcción de categorías y grillas adecuadas, como lo incierta que tantas veces resulta la ubicación de un artículo en alguno de los casilleros, que se hizo más delicada todavía con la intervención de los propios autores a través de las palabras clave.

Así, la exposición de tales problemas y sus posibles soluciones es un ejercicio frecuente. Alan Knight lo llevó a cabo en referencia a Past $\mathcal{E}$ Present (Knight, 2013: 80,81 y 82); Eduardo Míguez lo esbozó al momento de analizar el lugar de la historia social en la historiografía argentina a través del estudio de revistas (Míguez, 2008: 55,56), y se insinúa también por detrás de la reelaboración que Nicola Gallerano realizó sobre la grilla que había propuesto Darnton en 1980 para los Estados Unidos (Gallerano, 1989: 31,32). La organización de un sistema de casilleros eficaz y preciso para instalar los artículos no es, entonces, una operación sencilla, y los desajustes son difíciles de evitar. A ello se suman las pequeñas decisiones que deben tomarse para recoger los datos, cuyos fundamentos no pueden ser más que cualitativos, también expuestas por quienes se aproximan a estos asuntos; así ocurre, entre otras, con la decisión de tener o no en cuenta los artículos que componen cada dossier y los números especiales, por ejemplo.

Más allá de la cuestión de cómo tratar y organizar los contenidos de las publicaciones aparece otra, que incluye la relación que se supone tiene la revista con su contexto de producción más amplio. La revista académica de historia, o un conjunto de ellas, ha sido concebida a veces como un espejo, o un baremo según otros autores, de las tendencias de la historiografía nacional, y hasta internacional, en determinada coyuntura. Hay quienes han entendido más pertinente considerarla

2 Al parecer, del artículo de Corbin existen dos versiones previas, una publicada en Cahiers de l' Institut d'histoire de la presse et de l' opinión, de 1976, y otra del coloquio de 1979, que finalmente daría título al libro Au Berceau des Annales, publicado en 1983. A su vez, Robert Darnton presentaba sus cuadros de análisis de varias revistas americanas en 1980, en la compilación de Michael Kammen (ed.), The past before us. Contemporary Historical Writing in the United States. Darnton incluyó este trabajo en The kiss of Lamourette, cuya primera edición inglesa es de 1990. La edición castellana, del Fondo de Cultura Económica, apareció en Buenos Aires en 2010. Sobre la propuesta original, Nicola Gallerano propuso una adaptación en "¿El fin del caso italiano? La historia política entra la politización y la ciencia”, aparecido en Movimento Operaio e Socialista, Génova, año X, 1-2. 1987 y traducido al castellano en 1989. 
una ventana que permite asomarse a aquella otra producción de fondo que, en definitiva, la alimenta. Estas concepciones suelen compartir la idea de que los artículos de una revista, tomados en conjunto, deben ser considerados indicios particularmente significativos de aquellas tendencias. Sin embargo, la observación de algunas circunstancias mueve a la cautela. En principio, esas huellas pueden encontrarse también en otras producciones, ciertamente vinculadas a etapas previas de la formación profesional, como tesis de grado o posgrado y ponencias en jornadas y congresos; los libros, más propios de etapas posteriores, también podrían, o deberían, ser considerados. Por fuera de las asignaturas obligatorias en la universidad y otras instancias de formación superior, a su vez, la oferta y la demanda de seminarios permitirían del mismo modo acceder a información acerca aquellas grandes orientaciones. Nada garantiza que los resultados que pudieran obtenerse de la indagación de estos conjuntos documentales -que en principio permitirían construir una imagen más amplia- vayan a estar alineados con los que se alcancen a través un estudio que tome a las revistas, o a una de ellas, como fuente exclusiva. ${ }^{3}$

Finalmente, conviene recordar que ciertas características de las publicaciones suponen mediaciones, a veces muy importantes, entre aquella producción historiográfica de fondo y el conjunto de artículos publicados. Alcanza en este punto con señalar que las revistas de historia, pocas, que no fijan límites en cuanto a las áreas geográficas, las etapas, o las especialidades a las que deben ajustarse los artículos, parecen en principio una vía de entrada más vasta, y quizás más fiel, al estado general de la producción historiográfica y a sus tendencias.

A la luz de estas consideraciones, se observa que el Boletín exhibió algunas características peculiares que se han mantenido a lo largo de estos treinta años. En principio, que de las restricciones señaladas más arriba, la publicación solo asumió formalmente la que indicaba que se trataba de una revista de historia argentina -denominación habitual que, más allá de su pertinencia, remite a los procesos que tuvieron lugar en el actual territorio nacional- y americana, a la que estuvo dedicado un número notoriamente menor de artículos. A su vez, es sabido que aquí, como en otros ámbitos, gran parte de la producción historiográfica está dedicada a cuestiones que pueden instalarse en la historia nacional. Ese hecho no debe ocultar que el recorte excluyó otras especialidades que quizás logren exhibir una producción sólida y presencia internacional. Tales aspectos han de tenerse en cuenta al momento de apreciar cuánto dice de la coyuntura historiográfica una revista especializada que sea considerada aisladamente.

El trabajo sobre el Boletín resulta útil para percibir otros fenómenos. A pesar de que no existieron más restricciones formales que la indicada, una imagen del Boletín que suele estar asentada en muchos círculos académicos hace de él una revista centrada en procesos que, aproximadamente, ocurrieron entre el fin del período colonial y las primeras décadas del siglo XX; por otra parte, le asigna una

3 Un análisis que incluye, junto a las publicaciones, algunas de estas otras variables para el caso argentino en fechas recientes puede encontrarse en el trabajo de Martha Rodríguez (2003). 
especial predilección por la historia económica y social. Como todas, esa imagen tiene una relación equívoca y compleja con la realidad, aún si es eficaz, como en este caso. En cuanto a las especialidades, por ejemplo, tal representación no logra dar cuenta del peso creciente, en las páginas de la revista, de la historia política. Pero, por otra parte, si alguien atribuyera sin más al Boletín aquella condición de espejo de la producción historiográfica, o incluso de ventana, estaría impedido de observar el crecimiento importante y sostenido que ha experimentado la historia reciente en la Argentina, al menos desde hace 20 años e incluso algo más; la historia reciente ha hecho pie en la publicación sólo en los últimos años, y de manera todavía acotada. La expansión de la historia cultural e intelectual, un sector muy activo de la producción historiográfica, tampoco ha tenido un impacto evidente en las páginas de la revista. A estos puntos se volverá más adelante.

En esta ocasión, los números del Boletín serán vistos, por una parte, como un conjunto documental que permite percibir ciertas, sólo ciertas, características de la producción historiográfica argentina, en lo que hace a especialidades, problemas y períodos frecuentados. También han quedado en esa serie huellas de los cambios en las condiciones de producción historiográfica generales, así como de transformaciones ocurridas en el contexto más próximo del Boletín, esto es, en el propio Instituto Ravignani. Y, finalmente, se utilizarán algunos datos para observar el destino de propósitos y prácticas que la revista anunció al comienzo de la Tercera Serie, así como los cambios que tuvieron lugar en otros planos a lo largo de estos treinta años.

\section{Una revista universitaria de historia}

Como muchas otras publicaciones académicas y científicas del área de historia durante los siglos XX y XXI, el Boletín fue una publicación asociada a una institución universitaria, la Facultad de Filosofía y Letras de la Universidad de Buenos Aires; allí funcionó el Instituto de Investigaciones Históricas, luego denominado “Emilio Ravignani”, que en 1922 comenzó a publicar el Boletín. ${ }^{4}$ En los años que se estudian aquí, hubo en esa dimensión dos cambios relevantes: el instituto Ravignani se transformó en instituto de la Universidad de Buenos Aires, en 2007, y luego se produjo su incorporación al CONICET, como Unidad Ejecutora de Doble Dependencia, en 2011. Ambos cambios tuvieron lugar durante la dirección de José Carlos Chiaramonte (1986-2012).De todos modos, la vida cotidiana, tanto académica como administrativa, no dejó de estar vinculada centralmente a la Facultad de Filosofía y Letras; la incorporación de personal proveniente de CONICET fortaleció unos vínculos que, en la etapa anterior, se restringían a investigadores y becarios, tendían a ser individuales, y a veces se limitaban a una inclusión apenas nominal del instituto como lugar de trabajo.

4 Al respecto, ver el artículo de Pablo Buchbinder, "Los orígenes del Instituto de Investigaciones Históricas de la Facultad de Filosofía y Letras", publicado en este mismo número. 
En la Argentina, el anclaje en la base institucional de la universidad pública y del CONICET tuvo efectos de distinto tipo en el largo plazo. Por una parte, se cuentan las limitaciones económicas y financieras, manifiestas también en períodos anteriores, que en lo que hace a la Tercera Serie del Boletín se tradujeron ocasionalmente en retrasos importantes en la aparición de algunos números. A pesar de que hubo momentos menos complicados, el financiamiento fue una cuestión más o menos permanente, que la transformación en instituto universitario y luego en unidad de doble dependencia atenuó en parte, en una situación siempre condicionada por la marcha de variables macroeconómicas y por las cambiantes políticas científicas. Al aludir a la ubicación del Instituto y su Boletín en la estructura universitaria y científica, resulta útil advertir que no todas las revistas de historia locales cuentan o han contado con esa inserción, y que para ellas y los grupos que las impulsaron la situación fue todavía más precaria. Sin contar con base empírica suficiente, es posible sin embargo conjeturar que esas condiciones más duras pueden haber alentado, en otros emprendimientos, algún sentido de iniciativa colectiva y grupal, que en el caso analizado parece ser un rasgo menos acusado.

Por otra parte, la mencionada inserción hizo que el Boletín y el instituto estuvieran afectados por los procesos amplios que tuvieron lugar en la universidad y el sistema científico en general. Luego del fin de la última dictadura en 1983, paulatinamente y a pesar de algunas crisis económicas y políticas severas, se estableció un nuevo modo de relación entre el mundo académico y la coyuntura estrictamente política más próxima. Las mediaciones entre uno y otro mundo aumentaron, en un proceso que, según se ha planteado, deviene al mismo tiempo en el establecimiento de más distancia y en la emergencia de mayor especificidad en las disputas propias del espacio universitario y científico. Si se prefiere la fórmula, se amplía en parte una autonomía que, sin embargo, continúa siendo relativa.

Al mismo tiempo, la historia llamada profesional y sus instituciones, tan entramada con la de base universitaria, creció tendencialmente en el período. Como se verá, no se trata de un crecimiento explosivo ni de uno que sea el resultado de un reclutamiento más eficaz de estudiantes por parte de la historia a expensas de otras disciplinas. Es, en cambio, un crecimiento lento, que se aprecia mejor si se consideran las cifras absolutas. Así, más allá de los vaivenes de la economía, la continuidad institucional en la universidad y el sistema de investigación, sin cierres ni intervenciones, expulsiones de personal, exilios, prisiones o desapariciones, contribuyó a sostener aquel proceso, impactando en el primer movimiento en el número de estudiantes. El crecimiento de la matrícula termina por hacer aumentar el número de auxiliares docentes y profesores; a su vez, la demanda de profesores secundarios, cubierta en parte por la universidad y también por los profesorados, presiona sobre el número de carreras universitarias de historia, asociado al mismo tiempo a la creación de nuevas universidades, que se aceleró en el período. La organización de unidades de investigación, inicialmente dotadas de recursos escasos, acompaña el fenómeno con frecuencia. Algunas cifras permiten percibir estos cambios: en la Facultad de Filosofía y Letras de la Universidad 
de Buenos Aires, ámbito de pertenencia inicial del instituto, los alumnos de la carrera de Historia eran 1.602 en 1989, año en que comenzó la publicación de la Tercera Serie del Boletín, y 2.959 en 2015, última información a la que se accedió. Los graduados, también importantes para estos argumentos, crecían de 34 en el año 1989 a 86 en 2015, siempre en la UBA. En el conjunto de las universidades nacionales, las carreras de historia, en 2010, convocaban a 18.855 estudiantes, mientras que en 2015 las cifras se estiraban a 19.982. Hacia 2015, las carreras de historia en universidades estatales eran 30, algunas de ellas de creación muy reciente. ${ }^{5}$ En CONICET, por su parte, los investigadores en Ciencias Sociales y Humanidades, que incluyen los dedicados a la historia, pasaron de 1.008 en 2007, a 2.443 en 2019. En el mismo período, el número de becarios del área creció de 1.552 a $2.565 .{ }^{6}$ De zonas de ese universo en expansión moderada pero continua, provienen la mayoría de los autores que publicaron en el Boletín en el período, ya que la presencia de extranjeros no fue muy notoria. En cuanto al público, más adelante se señalarán algunos cambios importantes ocurridos en los últimos tiempos, pero puede plantearse con certeza que también allí, entre becarios, investigadores, profesores y estudiantes, se ubica casi el total de los lectores locales de los artículos de la revista.

También el propio Instituto Ravignani fue escenario de algunos de estos cambios. Tal como se indica en el artículo de Nora Pagano publicado en este número, en la primera mitad de 1966 el personal docente o de investigación llegaba a los 5 miembros. Ese mismo año, los grupos eran 3, con muy pocos integrantes y una denominación equívoca, que también ha subrayado Pagano: "cátedras o grupos de investigación”. Si se atiende en cambio a la coyuntura posterior al fin de la dictadura, se observa que en torno a 1987 eran cuatro los investigadores allí radicados. Hoy en día, el instituto reconoce 84 investigadores formados y 58 en formación, la mayoría de ellos agrupados en 5 programas y 8 grupos de investigación; es al mismo tiempo sede de proyectos acreditados por diversas instituciones. En cuanto al personal de apoyo a la investigación, biblioteca y archivos, el aumento es también notable. Así, en parte por efecto del proceso mencionado más arriba, en parte por su sólida presencia en la estructura de la historiografía universitaria, hecho que genera demandas por radicar allí investigaciones o becas, el instituto ha crecido sostenidamente, en particular en los últimos 15 años. Sin embargo, no se trata de un cambio sólo cuantitativo; acompasado con él, y alentado por la transformación en instituto de la UBA y la posterior incorporación a CONICET, se formalizaron aún más reglas y procedimientos, se ampliaron, se afinaron y se difundieron los requisitos para las incorporaciones y se crearon nuevos organismos que acompañan a la dirección, cuyos integrantes son elegidos por voto de los investigadores. Una situación diversa, por ejemplo, de la de 1966.

Retornando al Boletín, a lo largo de la Tercera Serie tuvieron lugar algunas transformaciones. En el Consejo Editor, naturalmente, han cambiado algunos nombres

5 Las cifras, en los correspondientes Anuarios de Estadísticas Universitarias, Ministerio de Educación, Secretaría de Políticas Universitarias.<Información disponible en el sitio: https://www.argentina.gob.ar/ educacion/universidades/informacion/publicaciones/anuarios

6 Recuperado de: https://cifras.conicet.gov.ar/publica/detalle-tags/017 
a lo largo del período por razones múltiples, y ha aumentado el número de integrantes respecto del inicial; el método de trabajo se ha mantenido a grandes rasgos. El Comité Consultivo es, en cambio, una creación reciente, fruto de alguna de las exigencias de la indización. Como es usual, ese consejo tiene una función que puede llamarse protocolar; forman parte de él historiadores externos en la actualidad al instituto, y muchos del exterior. Quienes ocuparon el cargo de Secretario de Redacción, y más adelante, los Asistentes de Redacción, fueron figuras decisivas a lo largo de los años de la Tercera Serie, tanto en las tareas más permanentes que reclama la publicación de una revista como en ocasión de las reuniones del Comité Editor. ${ }^{7}$

El Boletín, como artefacto cultural, exhibió gran continuidad. Desde el punto de vista material, el tamaño y las características de las tapas se mantuvieron y la cantidad de artículos publicados por número tendió a estabilizarse en los últimos años en 4, mientras que el promedio es de 3,6. A partir del número 2, se incorporó una sección llamada Notas y Debates, que ha aparecido en estos treinta años de modo intermitente. En el número 42, de enero-julio de 2015, el director Jorge Gelman anunció la decisión de comenzar a publicar números que incluyeran un dossier temático y de dar más impulso a la sección Notas y Debates. Una novedad de la última década, aproximadamente, fue la publicación de números especiales, dedicados a la Revolución de Mayo en el Bicentenario (2011), a José Carlos Chiaramonte (2016) y a Tulio Halperin Donghi (2018).

\section{Grandes tendencias: transformaciones, reorientaciones, permanencias}

Algunos autores, en trabajos aparecidos previamente, tomaron al Boletín como objeto de estudio, en ocasiones formando parte de un elenco de revistas académicas de historia. Así, en 1994 apareció el artículo de Nora Pagano y Pablo Buchbinder, en 2001 el de Oscar Aelo, y en 2008 el trabajo de Eduardo Míguez. El primero cubría los primeros 6 ó 7 números. El segundo, por su parte, tomó hasta el número 17, correspondiente a enero-junio de 1998. Finalmente, el artículo de Míguez se extiende un poco más allá de mediados de la década abierta en $2000 .^{8}$

Si bien aquellos autores se plantearon objetivos diferentes y centraron su atención en asuntos que no coinciden por completo, ni entre sí ni con los atendidos aquí, todos han ensayado la cuantificación de algunas variables, ejercicio cuyos límites hemos señalado más arriba. Así, a los problemas mencionados se añade en esta oportunidad el de intentar comparar datos recogidos en la actualidad y miradas que pueden abarcar unos treinta años, con información previa que ha

7 La lista completa de quienes formaron parte del Comité Editor a lo largo del período en diferentes momentos es la que sigue: Juan Carlos Korol, Luis Alberto Romero, Enrique Tandeter, Oscar Terán, José Luis Moreno, Fernando Devoto, Jorge Gelman, Noemí Goldman, Lilia Ana Bertoni, Ana María Presta, Hilda Sabato, Pablo Buchbinder, Claudio Belini, Roberto Schmit y yo mismo. Los Secretarios de Redacción fueron Noemí Goldman, Jorge Gelman, Roberto Schmit, Pablo Buchbinder, Julio Djenderedjian. A su vez, los Asistentes de Redacción fueron Julio Djenderedjian, Silvina Cormick y Juan Luis Martirén.

8 Los datos completos de las publicaciones figuran en la Bibliografía. 
sido obtenidos con otras herramientas y preguntas, y en otros momentos de la trayectoria del Boletín. Mirada la Tercera Serie desde una coyuntura diversa de las anteriores, entonces, se imponía un examen global, que considerara los 32 años de la revista, a pesar de la existencia de aquellos antecedentes. Se observará, de todas maneras, que alguna información ofrecida en los trabajos previos ha sido utilizada, y que tendencias percibidas allí han sido reconocidas o matizadas en este trabajo.

Desde 1989 y hasta la segunda mitad de 2020, el Boletín publicó 53 números regulares, a los que se suman los tres números especiales mencionados. La aparición semestral, estabilizada en los últimos tiempos, tuvo interrupciones importantes, vinculadas como se indicó a dificultades económicas: los dos primeros números fueron, de hecho, anuales, a pesar de las intenciones de los editores; el primer año en que apareció un número por semestre fue 1991. En 1997/1998, los números 16 y 17 aparecieron en un solo volumen y entre el número 25 y el 26 transcurrieron unos dos años. Las fechas de publicación indicadas en la revista y las reales, como ocurre tan a menudo, no suelen coincidir, aunque la situación se ha regularizado en los últimos años, favorecida por la existencia de la versión digital, presentada en 2012, cuando se lanzó un volumen que incluía los números 35/36. En esos 53 números se han registrado 193 artículos, sin considerar la sección Notas y Debates, ni tampoco los números especiales ni los artículos que integraron cada dossier, el primero de los cuáles apareció en el número 42, de enero-julio de 2015. La incorporación de esos materiales, en razón de sus características, podía interferir en el intento de percibir ciertas tendencias temáticas, de enfoque y de período analizado, si es que se tomaba al Boletín como una entrada, aún limitada, a zonas de la producción historiográfica dedicada a la historia argentina y americana. Merece señalarse, a su vez, que la sección Notas y Debates albergó, alentadas por el título, intervenciones importantes de autores locales y extranjeros que se permitían un tono más duro y polémico que el habitual en los artículos. En la sección Reseñas, la crítica ruda no fue el patrón dominante; por su parte, Nora Pagano y Pablo Buchbinder señalaron que allí se había reseñado una gran cantidad de libros publicados en el exterior, y también obras dedicadas a períodos que, en los artículos, no fueron particularmente atendidos; esa tendencia ha continuado hasta hoy (Pagano y Buchbinder, 1994: 114).

Vistos en estos treinta y dos años, con el cuadro que se ha utilizado aquí (Historia Económica, Historia Social, Historia Política e Historia Cultural e Intelectual; una opción clásica y sin sorpresas), se observan varios movimientos. Los trabajos que pueden ubicarse en el área de Historia Política, por ejemplo, crecen de un 25\% del total de 20 artículos que cubren casi por completo los cuatro números iniciales, a un $44,6 \%$ si se considera toda la serie. Una primera coyuntura de aumento consolidado y notable se ubica en la segunda mitad de los años noventa, cuando alcanzó un 40\%; luego, la tendencia continúa con alguna oscilación, hasta llegar a un 50\% hacia mediados de la década abierta en 2000 y a un estruendoso $75 \%$ en torno a 2010. Los temas y períodos abordados en los artículos que aparecieron en los años cercanos a 2010, contra lo que podría suponerse, no autorizan a pensar en la existencia de un "efecto bicentenario". A pesar de que un intento de 
este tipo abre demasiados flancos, ya que como se insinuó las grillas utilizadas no son las mismas ni pueden imaginarse idénticos los criterios para distribuir los artículos, se advierte que este panorama es parcialmente consistente con otros disponibles. Así, Pagano y Buchbinder registraron en 1994 un 20\% de artículos correspondientes a la historia política entre 1989 y 1993 (Pagano y Buchbinder, 1994: 113); Eduardo Míguez, a su vez, consideraba en 2008 que el 25,5\% correspondía a esa especialidad entre 1989 y 1995 (Míguez, 2008: 57). Con una grilla mucho más desagregada, lo que podría explicar las diferencias, y atendiendo exclusivamente a los autores argentinos, Aelo otorgaba a la Historia Política un 12\% hasta 1998 (Aelo, 2001: 105).

La Historia Económica, a su vez, experimenta una disminución importante, que la lleva de un 30\% a comienzos de los años noventa, a un $14 \%$ si se toman los años 2019-2020; para toda la serie, se obtiene un 19,2\%. A problemas de Historia Social, por su parte, estaba dedicado el $30 \%$ en los primeros números, entre fines de los años ochenta y comienzo de los noventa. En los números publicados a lo largo de 2019-2020, había descendido a 14,3\%. Si se toman en cuenta, en cambio, los 53 números, el porcentaje es de 20,2\%, en un declive semejante al de la Historia Económica. ¿Resulta prudente, dada la alta especialización y el hecho de que un ejercicio similar podría realizarse también con otras áreas, utilizando categorías híbridas, considerar la Historia Social y la Económica en conjunto, como hubiera sido pertinente y útil en otras etapas historiográficas, dado que ambas podían pensarse rivales de la Historia Política y alternativas a ella? El ejercicio no parece demasiado osado, pero tampoco demasiado perjudicial; las cifras que se obtienen indican un $60 \%$ para ambas especialidades a comienzos de los años noventa que desciende a un 34,5\% hacia 2019-2020; teniendo en cuenta toda la serie, las cifras rozan el 40\%, algo por debajo del porcentaje que corresponde a la Historia Política. Aún consideradas en conjunto, las huellas de la disminución resultan evidentes.

Para completar el cuadro, debe indicarse que la Historia Cultural e Intelectual se mantiene: de $15 \%$ en los primeros números a un $16,1 \%$ en la serie completa. En los años que van de 2016 a 2020 ha fluctuado entre el 30\% y el 35,7\%, lo que parece sugerir la consolidación de una tendencia al aumento, quizás más tardía que en el conjunto de la producción historiográfica local, aunque sería imprescindible un estudio en regla que corroborara esta impresión.

Otro aspecto que suele atenderse en este tipo de aproximaciones es la etapa a la que están dedicados los artículos; tampoco una periodización eficaz para la organización del material es sencilla de plantear. Las periodizaciones de la historia argentina que podemos suponer clásicas, cercanas a las que asumen a menudo las asignaturas universitarias, no acostumbran a lidiar con el mencionado punto de la pertinencia de la denominación para ciertas etapas. Por otra parte, ellas tampoco se alinean ajustadamente con las de otras naciones americanas, ni con la hispanoamericana tomada en conjunto, salvo en un nivel muy alto de generalización. Al mismo tiempo, muchos de los artículos desafían esos límites temporales, de base político-institucional, trabajando cuestiones que los desbordan. En 1994, Pagano 
y Buchbinder sostuvieron que al período posterior a 1930 estaba dedicado el 5\% de los artículos de aquellos primeros números (Pagano y Buchbinder, 1994: 114). A su vez, Aelo, tomando en cuenta los autores argentinos y utilizando otros cortes, planteaba que hasta 1998 el 12\% de los trabajos tomaban el período 1914-1955, mientras que el siguiente, que iba de 1955 hasta 1998, no contaba con ninguna contribución (Aelo, 2001:104). Así, el porcentaje de artículos sobre el período posterior a 1930 era bajo,y todavía más el de los de los dedicados al período abierto en 1945 y los siguientes. Tomando en consideración la serie completa, el porcentaje de estudios que trataron procesos posteriores a 1930 ha subido al 18,7\%. Si se recorta de ese porcentaje el de los artículos dedicados la etapa posterior a 1945, se alcanza el $8,1 \%$. Por otra parte, la historia colonial ha perdido peso frente a los temas de siglo XIX posteriores a 1810, y del siglo XX.

En lo que hace a los períodos más próximos de la historia argentina, y estos argumentos valen en principio también para la latinoamericana, es evidente que el Boletín no ha sido uno de los actores del proceso de "sostenida expansión numérica de las investigaciones sobre el pasado reciente en la Argentina, en el marco de una creciente institucionalización del campo de la Historia Reciente" (Franco y Lvovich, 2017:196), que los autores, en un trabajo publicado en la propia revista, fechan en torno al cambio de siglo, en un movimiento iniciado a fines de los años noventa. Es probable que ese rasgo del Boletín esté cambiando en los últimos años, pero es todavía pronto para afirmar si esa tendencia se estabilizará. Parece tratarse de una evidencia más de los límites de hacer de una publicación, en soledad, un espejo de la producción historiográfica, e incluso en una ventana: vista desde el Boletín, aquella expansión de la historia reciente prácticamente no habría tenido lugar.

En lo referido al escenario geográfico de los asuntos indagados en los artículos, el predominio del área rioplantense y del territorio que luego fue el de la Argentina es notoria desde el comienzo. Los artículos correspondientes al resto de Hispanoamérica constituyen un 13,5\% en el conjunto de la Tercera Serie.

Finalmente, se puede echar una mirada a las filiaciones institucionales de los autores. Luis Rodríguez Yunta indicó, en referencia al Anuario de Estudios Americanos, revista del CSIC español, que "aunque las dos principales instituciones representadas en el Anuario de Estudios Americanos, Universidad de Sevilla y CSIC, destacan con claridad sobre las restantes, el índice de endogamia es muy bajo". Sobre el total de artículos "se aprecia que entre ambas no superan el $30 \%$ de los trabajos publicados, y no llega en ningún periodo al 15\% para el caso de la entidad editora (CSIC).El porcentaje de artículos firmados por investigadores de la entidad editora en Anuario de Estudios Americanos para el total del periodo analizado es de solo el $8 \%$, el más bajo entre las revistas españolas de Historia de América que se han analizado en este trabajo" (Rodríguez Yunta, 2018: 744 y 745).

En ambas citas se insinúan dos problemas que atañen al Boletín, pero que tienen una dimensión más amplia: qué entender por “entidad editora”, y qué considerar alto o bajo en cuanto al índice de endogamia, siendo que no se han 
revisado otras publicaciones argentinas en esta ocasión. En cuanto a la segunda cuestión, al menos para el caso español que se ha citado, un 15\% es reputado como bajo, y al parecer también un $30 \%$. La primera cuestión es más delicada; para obtener las cifras que se plantearán, se utilizó un criterio que indica que la "entidad editoria” es el Instituto Ravignani, no la Facultad de Filosofía y Letras, ni tampoco la Universidad de Buenos Aires o el CONICET. Ello obedece a que, a diferencia de lo que ocurre en otros sistemas nacionales de investigación, la sociabilidad académica, las reuniones de trabajo y, fundamentalmente, la toma de decisiones sobre la revista, tienen lugar en los institutos, no en aquellos otros organismos. Resulta algo forzado considerar como autor "interno" al Boletín a un investigador de CONICET con lugar de trabajo, por ejemplo, en un instituto ubicado a cientos de kilómetros de la sede del Instituto Ravignani, o a un investigador de otro instituto de la Facultad de Filosofía y Letras. Utilizando estos parámetros, la revista se muestra como una publicación poco endogámica y abierta a un conjunto amplio de investigadores: de los autores de artículos cuya filiación institucional se pudo establecer, aproximadamente un 22 \% eran miembros del Instituto Ravignani al momento de publicar. Utilizando una perspectiva semejante, Pagano y Buchbinder habían indicado en 1994 que "sólo en un 27\%" los autores de los artículos de los primeros números pertenecían al instituto, hecho que contrastaba con lo que ocurría en otras publicaciones (Pagano y Buchbinder, 1994: 114). ${ }^{9}$ Con otros criterios, que suponen agregar a los investigadores del instituto los de CONICET, Aelo ha señalado, que hasta 1998, el 25 \% de los autores argentinos eran miembros del Instituto Ravignani (Aelo, 2001: 109)

\section{Un cambio con orígenes externos, un cambio crucial, un cambio todavía en curso}

Según sostienen los especialistas, los intentos de creación de bases de datos que permitieran identificar el conjunto principal y más representativo de las revistas de cada disciplina tuvieron, a mediados de los años treinta, un primer capítulo importante, con la construcción de un modelo matemático, la llamada ley de distribución de Bradford, que permitiría a los bibliotecarios detectar ese grupo teniendo en cuenta las demandas de los usuarios. Años más tarde, en torno a 1960, Eugene Garfield, a cargo del Institute for Scientific Information, una entidad privada, utilizó el modelo en las varias bases de datos que la institución empezó a publicar un año más tarde; en 1973 se publicó el Social Science Citation Index y en 1978 el Arts $\mathcal{E}$ Humanities Citation Index. Por entonces, comenzó a utilizarse otra herramienta en el análisis, que apreciaba las citas registradas de revistas y artículos: el factor de impacto. ${ }^{10}$ Es posible

9 Diferentes criterios se utilizan en las indizadoras en las que el Boletín está incluido y es evaluado. La Red de Revistas Científicas de América Latina y el Caribe, España y Portugal (Redalyc), por ejemplo, indica un porcentaje de endogamia mayor en las publicaciones del Boletín, porque considera como autores "internos o internos no institucionales" a quienes pertenecen a la Universidad de Buenos Aires o el Conicet.

10 Se sugiere, sobre estos asuntos, analizados en versión más detallada, la consulta del trabajo de Romanos de Tiratel y López (2004), y del de Rozemblum (2014), citados en la bibliografía. 
que allí se encuentren los orígenes lejanos de ciertos procesos recientes que terminarían afectando a las revistas científicas en general y con ellas, a las de historia.

Así, aquellas tendencias, enlazadas con las nuevas posibilidades que ofreció el soporte digital, impactaron en el Boletín, como venía ocurriendo con diferencias de ritmo en el horizonte internacional. Es en este sentido que se trata de cambios cuyos inicios están más allá de la propia revista, ya que fue esta una tendencia amplia y general entre las publicaciones académicas y científicas, a la que finalmente el Consejo Editor terminó por incorporar al Boletín. Ese proceso de cambio tuvo dos piezas centrales entramadas: la indización y la digitalización.

A fines del siglo XX y en los primeros años del XXI, se organizaron algunas bases de datos que trataron de enfrentar los que consideraban límites de las disponibles -el del idioma era uno, dado el predominio del inglés; el de la ponderación de la producción científica de las naciones y regiones periféricas, otro, por ejemplo. Así, entre 1994 y 1997/1998 se organizó Latindex y, en 1997, Scielo. En 1999 se puso en marcha un proyecto de CONICET radicado en el Centro Argentino de Información Científica y Tecnológica (CAICYT), hoy un instituto del Consejo, que devino en la constitución del Núcleo Básico de Revistas Científicas Argentinas. A su vez, en 2002 se presentó la Red de Revistas Científicas de América Latina y el Caribe, España y Portugal (Redalyc). El procedimiento de indización, en una versión despojada, consiste en la incorporación de una revista a alguna de las bases de datos a través del cumplimiento de ciertos requisitos que esta última determina; la operación suele culminar con una valoración cuantitativa fundada en el grado de cumplimiento de esas exigencias.

El Boletín del Instituto Ravignani fue indizado en Scielo en 2004 y en Latindex en 2006, luego de trámites que habían comenzado unos años antes. También en 2006 tuvo lugar la inclusión en el Núcleo Básico de Revistas Científicas Argentinas; el Portal de Publicaciones Científicas y Técnicas (PPCT, en adelante), también del CAICYT, creado en 2009, alberga las versiones digitales del Boletín desde 2012. A su vez, el Boletín presentó en 2012 el primer número digital, el 35/36. A partir de ese momento, la revista comenzó apublicarse en papel y en versión digital, en la mencionada plataforma virtual que ofrecía el PPCT.

Esa circunstancia, a la que se suma la posterior digitalización de los números anteriores de la Tercera Serie y su instalación en la página propia del Instituto y de las publicaciones de la Facultad de Filosofía y Letras, del PPCT y de Redalyc, con acceso abierto, produjeron cambios muy importantes para la revista, abriendo una etapa que sigue en curso.

Esas transformaciones no tuvieron lugar en el plano de los asuntos analizados en los artículos, ni en el de los períodos de los que se ellos se ocupan; tampoco en el sistema de evaluación de los trabajos, que continuó siendo externo y anónimo. Afectó, en cambio, lo relativo al contacto de la publicación con sus lectores, transformado radicalmente por la posibilidad, siempre que se cuente con internet, de 
acceso abierto e inmediato a cada nuevo número publicado, a los anteriores de la serie, e incluso, actualmente, a los de la Segunda Serie (1956-1982). ¿Cuáles son entonces algunos de esos cambios, que en ciertos casos están en ciernes, y en otros, más avanzados? De ellos se tomarán dos: las cifras de los que pueden presumirse lectores en la etapa digital, por un lado, y las posibles transformaciones en las prácticas de lectura, que no afecta solo, desde ya, a los públicos del Boletín. Como se advierte fácilmente, el último tendrá un tono conjetural.

En cuanto al primer cambio producido por la digitalización, el de los lectores y el público, sus huellas son visibles. Algunas estimaciones indican que, en la actualidad, las ventas de la versión en papel rondan los 25 ejemplares, cifra que revela la inclinación al acceso digital. También el canje con otras revistas ha decaído, en parte porque algunas de ellas han sido discontinuadas, en parte por inconvenientes financieros para el envío. La edición en papel es hoy de unos 60 ejemplares por número publicado. No se cuenta con datos firmes acerca de la situación previa a la aparición de la edición digital, en razón de la imposibilidad actual de consulta de algunos archivos.

Si por el contrario se presta atención a las estadísticas sobre las visualizaciones de los números publicados descle la aparición de la versión digital, el panorama cambia. Desde que se publicó el primer número digital, el 35/36, que corresponde a julio-diciembre de 2011 y enero-junio 2012, hasta el número 51 de juliodiciembre de 2019, los resúmenes de los artículos han recibido un promedio de 5.147 visualizaciones, y los artículos un promedio de 7.922. ${ }^{11}$ A esta situación debe sumarse la posibilidad de acceso al resto de los números de la Tercera Serie, que los mantiene disponibles para la consulta inmediata. Las herramientas que ofrece internet, a su vez, permiten obtener información sobre el público y, por ese camino, pueden contribuir a que la revista cuente con más datos sobre su circulación y, en definitiva, sobre aspectos del propio emprendimiento editorial que no eran accesibles y que forman parte central de él. ${ }^{12}$

Por otra parte, la segunda posible transformación, relativa a los nuevos modos de lectura y las prácticas que involucran, ha sido tratada en algunos trabajos, incluso por parte de autores que se dedicaron a indagar cómo esos procesos afectaban la edición de libros de historia; ante objetos tan difíciles de asir, suele tratarse de observaciones con apoyos empíricos discontinuos y parciales. Así, sostuvo Sophie Barluet en 2005 que "de ahora en más, leemos como circulamos en internet: de manera fragmentaria y dispersa” (Barluet, 2005: 83) ${ }^{13}$. Pero, aun si se considera que esta opinión es precisa, y es muy probable que lo sea, queda pendiente el problema de los tonos específicos que ese tipo de lectura adopta cuando se trata

11 Estos datos corresponden a las estadísticas de cantidad de visualizaciones que arroja el sistema Open Journal System (OJS) del portal del PPCT, que fue la página principal del Boletín entre 2012 y 2020 . Si bien se trata de la mayor parte de las consultas, es de destacar que no incluye las visualizaciones en otros portales que albergan la revista (como Scielo o Redalyc) o bien las páginas personales de autores (como por ejemplo, Academia.edu).

12 Se sugiere sobre el punto la consulta de los trabajos de Leticia Cerezo y Marcela Ferrari (2013), y del de Nicolas Roussellier (2014).

13 La traducción es del autor. Original en francés. 
de artículos altamente especializados, que incluso serán probablemente utilizados como bibliografía en nuevas investigaciones. En cualquier caso, se trata sin dudas de una cuestión aún abierta, cuyo tratamiento podría comenzar recordando que la misma incertidumbre acerca de la relación entre el acceso digital, la lectura efectiva y sus modos, existía, en la etapa anterior, entre la compra o consulta en biblioteca, la lectura efectiva y sus modos.

\section{5. ¿Un doble programa incial?}

Hacia 1995, Nicolas Roussellier señaló que en Francia, entre 1945 y esa fecha, en las publicaciones periódicas dedicadas a la historia fueron "raros" los textos "de manifiesto", en los que "las revistas hablan de ellas mismas" (Roussellier, 1995: 127) ${ }^{14}$. Tampoco fue esa una práctica ejecutada con intensidad en la Tercera Serie del Boletín: las intervenciones de la Dirección o del Comité se reducen a 3, además de la presentación del primer número. De ellas, dos anuncian la incorporación de nuevas secciones y una es una semblanza de Jorge Gelman, en ocasión de su muerte, ocurrida en 2017. Se cuentan además algunas presentaciones breves de los números especiales.

Sin embargo, en el primer número de la Tercera Serie, de enero-junio 1989, el director José Carlos Chiaramonte planteó en la "Presentación" varios argumentos en los que vale la pena detenerse. No es posible, con la documentación disponible, definir cuántos de ellos fueron compartidos o discutidos por los miembros del Consejo Editor; en consecuencia, serán en principio atribuidos al director, aunque se les podría adjudicar una representatividad mayor, ya que no hay manifestaciones de desacuerdos en la publicación. Chiaramonte señaló los que entendía eran rasgos del grupo de historiadores que se empeñaba en volver a publicar la revista. "Quienes emprenden esta nueva etapa”, sostenía, "pertenecen al conjunto de historiadores argentinos que desde la segunda posguerra en adelante ha tratado de contribuir a renovar la historiografía iberoamericana, incorporando, por una parte, los logros del desarrollo de la historia social y económica, y de la correspondiente renovación de la historia cultural y política”. No es difícil reconocer en esa caracterización a los grupos que por entonces comenzaban a denominarse "renovadores" en los análisis de la historia de la historiografía argentina, que habían actuado en algunos centros universitarios desde fines de los años cincuenta y comienzos de los sesenta, luego del golpe de Estado que derrocó al peronismo en 1955.

Al mismo tiempo, Chiaramonte planteaba para la revista el horizonte de un público "más amplio" que el especializado, "con la esperanza de contribuir a disipar equívocos que rodean asiduamente la producción historiográfica en los países latinoamericanos". Tales equívocos, entendía, tenían origen en la "tendencia simplificadora, generada en el terreno político", que se inclina a forzar "la 
interpretación del pasado para adecuarlo a la explicación de los problemas del presente”. Criticaba, además, "el anacronismo de moldear los conflictos históricos por proyección de los de nuestros días", que habría "hecho estragos en la historiografía latinoamericana". Esta tendencia "se ha extendido en la cultura histórica latinoamericana, e influye en buena parte de la labor universitaria”. Luego de observar que la "búsqueda de antepasados a los grandes partidos del presente, tiende a suponer conflictos del siglo XIX similares a los de nuestros días”, Chiaramonte retornaba a los anhelos del Boletín y añadía que "si una revista como esta pudiese contribuir a la mejor comprensión de conflictos históricos aún vivos en la actual cultura política latinoamericana, habría cumplido también con una de las más caras aspiraciones de sus editores”.

Más adelante, comunicaba que el Comité Editor "ha organizado un sistema de arbitraje con historiadores ajenos a él", con el objetivo de "buscar el mejor nivel para sus materiales". El arbitraje externo garantizaría, en esos argumentos, el "nivel científico" deseado (Chiaramonte, 1989: 5-6). Esa misma indicación fue reiterada en el segundo número.

El director, de este modo, asignaba al Boletín dos objetivos enlazados: constituirse en una revista que cumpliera con estándares científicos adecuados, que al mismo tiempo promoviera, en un público "amplio", una comprensión más acabada de conflictos históricos que entendía todavía activos en la "cultura política latinoamericana”. Esos propósitos estaban planteados de cara a un diagnóstico que indicaba que aquella tendencia "simplificadora" era visible en la "cultura histórica" -que puede suponerse una que no se limitaba a la historia universitaria o profesional-, pero también en la "historiografía latinoamericana" y en la "labor universitaria". El arbitraje, entonces, no sólo era una práctica que garantizaría la cientificidad de los artículos publicados, sino un instrumento en aquella otra tarea de promover una aproximación al pasado distinta a la que consideraba habitual en la cultura política regional, que debía emprender una revista científica.

Este modo de entramar unos objetivos que eran de orden científico, cuya consumación tendría lugar en el espacio académico, con otros que, obligatoriamente, sólo podrían alcanzarse más allá, no era nueva ni en la historiografía local ni en la internacional. Formaba parte, en cambio, de una larga tradición que se remontaba a fines del siglo XIX europeo, cuando menos, y al proceso de organización de la que suele llamarse historia profesional. En torno a estos puntos, basta mencionar unas pocas revistas extranjeras, con diferentes perfiles historiográficos e intelectuales, y desparejas presencias internacionales, que podrían ubicarse en un esquema sumario de objetivos de una revista de historia tradicional de fines del siglo XIX, planteos de Annales y posterior argumento de elencos que incluían a historiadores marxistas.

Así, en Francia, Gabriel Monod, al presentar la Revue Historique en 1876, luego de señalar que ella "será una publicación de ciencia positiva", sostuvo que las luchas políticas y las pérdidas territoriales de 1870 "convierten en un deber el despertar, en el alma de la nación, la conciencia de sí misma por medio del profundo 
conocimiento de su historia”. ${ }^{15}$ Un deber que, es evidente, habría de jugarse en el mundo político y social. Más adelante, Marc Bloch, miembro de los Annales, uno de los sectores adversarios de los grupos historiográficos en los que había formado Monod, identificó "dos problemas por resolver: el de la legitimidad intelectual de la historia corno disciplina científica (que pertenece a la línea de desarrollo de la historia-ciencia) y el de la utilidad de los estudios históricos para la sociedad (que pertenece a esa relación más amplia de los grupos humanos con el pasado [...])"(Mastrogregori, 1998: 97). Hasta la guerra al menos, Annales buscó públicos más allá de los especializados. A comienzos de la década abierta en 1950, por su parte, Past \& Present se presentó afirmando su convicción de que "la aplicación de la técnica científica construida por la investigación histórica del siglo XIX" no estaba invalidada. Apelando a Polibio, señaló compartir su creencia "en el valor de la historia para el presente", para agregar que "es a la historia a la que la gran mayoría de los hombres y mujeres pensantes miran en busca de fortaleza y entendimiento" 16 .

Más allá, entonces, del éxito o el fracaso de la empresa, y de que el propósito podría haberse planteado con tonos más épicos o más estridentes, el que el director diseñó para el Boletín no fue, en cuanto a intentar influir más allá de los límites del espacio académico y científico, un objetivo módico. Sin embargo, las intervenciones de este tipo, como se indicó, no se repitieron en el Boletín.

Por su parte, el arbitraje externo y anónimo a cargo de los llamados pares, ha conservado hasta hoy un lugar central en la revista; esa es la práctica que acostumbra destacarse cuando se expresan, formal e informalmente, caracterizaciones de la publicación. Acerca de él, Alan Knight, citando a Jack H. Hexter, señalaba que "afortunadamente, la historia, incluso la historia académica, suele estar - y debe estar- radicada en una realidad empírica y sujeta a esa 'institución central de la sociedad de historiadores... el juicio de sus pares' ", (Knight, 2013: 88). ${ }^{17}$

Puede, entonces, examinarse cuántos de aquellos anhelos que se expresaban en la presentación se han cumplido acabadamente. Como insinuamos, el del nivel científico, garantizado en aquellos razonamientos por el sistema de arbitraje, parece haber sido alcanzado. En cuanto al de intervención en el mundo político-cultural, la situación es otra. Es posible que a explicarla contribuyan varios factores. Uno de ellos es que a pesar de inscribirse en una tradición ya larga, se trataba quizás de un propósito excesivo para una revista científica, cuyas características materiales e intelectuales más relevantes no se modificaron; no parece esa una herramienta particularmente útil para aquella tarea, que no puede desentenderse del problema de los públicos. A su vez, las discusiones colectivas sobre el pasado, reciente o lejano, y también las políticas estatales y de partidos hacia él, tuvieron una marcada continuidad en el período, a pesar de alzas y bajas en su intensidad; se trató de un escenario poblado por muchos actores y muchas voces, en buena parte potentes.

15 La cita de Monod, en Bourde, G. y H. Martin (1992: 147-148).

16 En la presentación de Past \& Present, firmada por “The Editors", 1952, p. III. La traducción es del autor. Original en inglés.

17 La cita orignal en Hexter (1971: 82). 
Así, el Boletín resultó un instrumento adecuado y se demostró muy eficaz para cumplir con la dimensión académica del objetivo inicial, tal como demuestra la consideración alcanzada en el ambiente historiográfico, quizás más aún que las indizaciones. La otra tarea, puede pensarse, todavía continúa pendiente. 


\section{Bibliografía}

" Aelo, O. (2001). De historiadores e historiografía. Apuntes sobre el Boletín del Instituto Ravignani. Taller. Revista de Sociedad, Cultura y Política, vol. 6, nro. 16, pp. 95-118

" Barluet, S. (2005). L'édition en histoire: anatomie d'une crise. Vingtième Siècle. Revue d'histoire, pp. 81-89.

" Bourde, G. y H. Martin (1992). Las escuelas históricas. Madrid: Akal.

" Carbonell, Ch-O. (1983). L'analyse de contenu d'une revue historique: I'analyse quantitative. Storia della Storiografia, nro. 2, pp. 96-112

"Cattaruzza, A. (2018). Un siglo de libros de historia en la Argentina: la cultura, la política y el mercado editorial. Badebec, nro. 15, pp. 199-235

" Cerezo, L. y M. Ferrari (2013). PolHis. Una experiencia editorial en el contexto historiografico argentino de comienzos del siglo XXI. Anuario Colombiano de Historia Social y de la Cultura, vol. 40, núm. 1, 3, pp. 203-240.

"Chiaramonte, J. C. (1989). Presentación. Boletín del Instituto de Historia Argentina y Americana "Dr. Emilio Ravignani", nro. 1.

" Corbin, A. (1983). La Revue Historique. Analyse de contenu d' une publication rivale des Annales. Au Berceau des Annales. Toulouse: Presses de I'Institut d'études politiques de Toulouse

" Darnton, R. (2010). El beso de Lamourette. Reflexiones sobre historia cultural. Buenos Aires: Fondo de Cultura Económica.

" Gallerano, N. (1989). ¿El fin del caso italiano? La historia política entra la politización y la ciencia. Cuadernos de Teoría e Historia de la Historiografía, nro. 10

" Guédon, J-C.(2011). El acceso abierto y la división entre ciencia "principal" y "periférica". Crítica y Emancipación, nro. 6, pp. 135-180

" Hexter, J. (1971), Doing History. Londres: George Allen and Unwin.

" Franco, M. y D. Lvovich (2017). Historia Reciente: apuntes sobre un campo de investigación en expansión. Boletín Del Instituto De Historia Argentina Y Americana Dr. Emilio Ravignani, nro. 47, pp.190-217.

" Knight, A. (2013). Las revistas históricas y América Latina: una perspectiva europea/ inglesa. Anuario Colombiano de Historia Social y de la Cultura, vol. 40, núm. 1,3, pp. 6797.

" Marín Gelabert, M. (2001). AYER. Luces y sombras del contemporaneísmo español en la última década". Ayer, nro. 41, pp. 213-25.

" Mastrogregori, M. (1998). El manuscrito interrumpido de Marc Bloch. México: Fondo de Cultura Económica.

" Míguez, E. (2008). Entre la economía y la política: el espacio de la historia social en la historiografía argentina. En Mallo, S y B. Moreyra (eds). Miradas sobre la historia social en la Argentina en los comienzos del siglo XXI. Córdoba, La Plata: Centro de Estudios Históricos "Prof. Carlos S. A. Segreti" y Centro de Estudios de Historia America Colonial, pp. 49-64.

"Nisonger, T. (1998). Management of Serials in Libraries. Englewood, CO: Libraries Unlimited. XXVIII, $433 \mathrm{p}$. 
" Pagano, N. y P. Buchbinder (1994). Las revistas de historia en la Argentina durante la década de los ochenta. En Devoto, F. (comp.). La historiografía argentina en el siglo XX (II). Buenos Aires: CEAL, pp. 110-125

" Rodríguez, M. ( 2003) Una década de historiografía argentina (1990-2000). Orientaciones, temas y problemas. Anuario del Centro de Estudios Históricos "Prof. Carlos S. A. Segreti", nro 2-3, pp. 285-305

" Rodríguez Yunta, L. (2018). La revista Anuario de Estudios Americanos analizada desde las bases de datos documentales: evolución temática, distribución de la autoría e impacto. Anuario de Estudios Americanos, 75, 2, pp. 731-753

" Romanos de Tiratel, S. y López, N. (2004). Las revistas argentinas de historia: visibilidad en bases de datos internacionales. Información, cultura y sociedad, nro. 11. pp. 95-115

" Roussellier, N. (1995). Les revues d'histoire. En François Bédarida (dir). L'histoire et le métier d'historien en France 1945-1995. Paris: Édition de la Maison des sciences de l'homme, pp. 127-146.

" Roussellier, N. (2014). Les mutations de Vingtième Siècle. Revue d'Histoire. Internationalisation et numérisation. Vingtième Siècle. Revue d'histoire, nro. 124, 111-121

"Rozemblum, C. (2014). El problema de la visibilidad en revistas científicas argentinas de Humanidades y Ciencias Sociales: Estudio de casos en Historia y Filosofía. Tesis de posgrado. Universidad Nacional de Quilmes. Disponible en: http://www.memoria.fahce. unlp.edu.ar/tesis/te.1031/te.1031.pdf

" The Editors (1952). Introduction. Past \& Present, nro. 1, febrero 1952, pp. I a IV 